\title{
L'universe fantastique \\ de Jean Ray
}

\section{Virginia Boza Araya}

Universidad Nacional de Costa Rica

Lorsqu'on évoque la littérature belge d'expression française, on se réfère rarement à la littérature fantastique. Le présent article a pour but de présenter l'écrivain Jean Ray, un écrivain belge qui a marqué son époque avec d'étonnantes œuvres fantastiques, hélas méconnues et peu étudiées.

Jean Ray, de son vrai nom Raymond Jean Marie De Kremer, est l'un de grands maîtres de la littérature fantastique. Il est né en 1887, à Gand, où il a passé presque toute son existence. Il est mort en 1964.

De nombreux spécialistes le considèrent «l'Allan Poe» belge et de ce fait, l'un des rares à pouvoir rivaliser avec autant de maîtrise et d'aisance avec les maîtres anglo-saxons de ce genre qui leur est propre. A cet égard A. Dorémieux affirme que «Il n'y a que Lovercraft qui vous donne pareille sensation de démesure, qui communique à ce point le vertige».'

Son succès, il le doit non seulement au mélange de culture flamande et d'éducation francophone mais aussi au Ham l'un des quartiers les plus sombres et les plus vieux de sa ville natale dont l'atmosphère noire. froide, a fortement influencé sa conception du fantastique. Elle a inspiré à l'œuvre de Jean Ray le décor brumeux où règnent la terreur et une vision particulière de la mort.

1. André Dorémieux. "Littérature fantastique: Malpe'rnics par Jean Rạ̣"» e n Ficrion 18 (Paris: Editions OPTA, 1955) 112. 
Son œuvre est vaste. Elle comprend près de 190 contes et nouvelles fantastiques, d'horreur et d'épouvante et une trentaine où le fantastique se mélange à d'autres genres. Il aborde tous les genres: des poèmes, des chroniques artistiques, des critiques sur l'art et la littérature, des reportages, des revues théâtrales, des scénarios... Il a écrit aussi bien en français qu'en néerlandais, en anglais, en allemand en signant ses œuvres, sous des pseudonymes divers dont les plus connus sont Jean Ray et John Flanders. Il a été traduit en anglais, en espagnol, en italien, en portugais, en japonais. Jean Ray est l'un des plus grands novateurs de la littérature fantastique. Il renouvelle non seulement la poétique du genre mais aussi la thématique. Dans un premier temps je ferai référence aux aspects qui caractérisent son œuvre. Puis, une approche plus approfondie de sa création permettra de mieux illustrer sa conception du fantastique. Étudier une œuvre aussi considérable que celle de Jean Ray est un véritable défi étant donné la richesse et la diversité des thèmes qu'il exploite avec autant d'aisance les uns que les autres. Il développe une série de motifs qui vont marquer le fantastique moderne et donner plus de profondeur et de relief à des composantes, la femme par exemple, jusque-là peu ou pas du tout présentes.

\section{La modernité de l'œuvre}

La modernité se manifeste en tout premier lieu par le fait que les thèmes choisis par l'auteur belge constituent un univers fantastique très personnel où la quatrième dimension ${ }^{2}$ constitue l'épine dorsale. Contrairement au merveilleux qui place le monde surnaturel hors de la réalité humaine, le nouvelliste l'insère dans le quotidien, au cœur

2. D'autres termes qui désignent la quatrième dimension sont: Mondes parallèles. plans ou espaces intercalaires. espaces hy'pergéométriques. plans de l'hyperespace. Jean Ray a exploité le thème des mondes parallèles dans une vingtaine d'cuvres de fiction parmi lesquelles figurent ses grands chefs d'œuvre «Le Psautier de Mayence», «Le Grand nocturne» (1942). Malpertuis et “La Ruelle ténébreuse». Bien que n'étant pas le motif central, il l'évoque dans beaucoup d'autres ouvrages comme. par exemple. "Dürer l'idiot» (19.31), "L'Assıette de Moustier», "L'Auberge des spectres». «Le Miroir noir». 
même de la vie. C'est pour lui un espace inviolé, situé entre l'humanité et la divinité, où dieux et humains peuvent se rejoindre sur un même pied d'égalité. Cette conception découle de la passion qu'il éprouvait pour les mathématiques et la théorie de la relativité d'Einstein qui postule la notion de simultanéité de deux événements se produisant en des lieux différents. Cet attachement est évident dans «La Ruelle ténébreuse» et dans plusieurs de ses œuvres ${ }^{3}$ : «La science moderne n'est-elle pas aculée à la faible euclidienne par la théorie de cet admirable Einstein que le monde entier nous envie?» ${ }^{4}$

Cette théorie va étayer sa vision d'un univers hypergéométrique car elle démontre que tout objet, au-delà d'une première apparence, peut varier à l'infini selon la position de l'observateur. Cette relativité va marquer son œuvre à tous les niveaux et provoquera un bouleversement total du genre.

Autour de ces mondes intercalaires ou Quatrième Dimension, l'auteur réussit avec une grande maîtrise à entrelacer en parfaite harmonie des thèmes exploités par la science fiction tels que les mathématiques, la recherche scientifique, la contraction du temps et de l'espace avec les thèmes traditionnels du genre fantastique parmi lesquels peuvent être cités les fantômes, le diable et le pacte diabolique, la mort personnifiée, les vampires, les lycanthropes, et d'autres métamorphoses dues en particulier à la magie noire.

Ils sont si savamment reliés entre eux qu'ils construisent de nouvelle en nouvelle un univers singulier possédant ses propres lois, dimensions, principes, habitants... Marc Vuijlsteke le fait si bien remarquer:

3. "La Ruelle ténébreuse» en La gerbe noire (Paris: NEO. 1984) 148, «Le Psautier de Mayence», “Le Grand nocturne» (1942) en Le Grand Nocturne. Les Cercles de l'éponvante (Bruxelles: Actes Sud-Labor. 1989), “La Trouvaille de Mr. Swectpipe» en Visages et choses crépusculaires (Paris: NEO, 1982) 174, "Mathématiques supérieures» et «Le Tessaract» en Le Carrousel des maléficess (Verviers: Marabout. 1964).

4. "La Ruelle ténébreuse», 148. 
tous les thèmes et motifs de Jean Ray sont étrangement reliés les uns aux autres, de sorte qu'ils semblent faire partie d'un plus grand ensemble, d'une option fondamentale et organisatrice structurant la totalité de l'œuvre. ${ }^{5}$

Son univers est bâti autour d'un espace hypergéométrique caractérisé par une série de plans intercalaires, superposés ou parallèles où éléments et créatures sont d'une grande cruauté. Ces lieux seraient invisibles et impénétrables (en apparence): Il existe un monde voisin, invisible, impénétrable pour nous, parce qu'étant situé sur un autre plan. Ce monde est étrangement, criminellement... réuni au nôtre. ${ }^{6}$

En fait, il y a plusieurs façons d'y accéder. Dans bien des cas, la découverte des mondes parallèles est l'œuvre du hasard. Dans Malpertuis, le marinier Anacharsis, poussé par le vent, arrive à une île où se trouvent les restes des dieux grecs. Le héros du conte «La Grande ourse», lui aussi, fait une incursion dans un plan intercalaire, lors d'une petite promenade dans un quartier sale et lugubre de Londres. Il se trouve, sans le savoir, en présence d'un mort. Quand au protagoniste de «La Trouvaille de Mr. Sweetpipe» il découvre par hasard la clé du passage à cet autre plan de l'espace en essayant de compléter des formules algébriques inachevées, trouvées sur un feuillet. C'est sa passion pour les calculs, les équations, les logarithmes qui va le précipiter dans cette autre dimension. Dans d'autres cas les personnages cherchent à y pénétrer grâce à des formules mathématiques, à des observations scientifiques, à des formules magiques. ${ }^{7}$ Certains de ces mondes parallèles sont inconnus, mystérieux, indescriptibles en termes

5. Marc Vuijlsteke. "Les Univers intercaires de Jean Ray" en Les. Cahiers de L'Herne (Paris: L'Herne. 1980) 234.

6. Jean Ray, "Les Etranges Etudes du Dr. Paukenschlänger» en Les Contes du whisky (Paris: Librairie Champs Elysées. 1980).

7. "La Grande ourse». Les contes noirs du Golf (Verviers: Marabout Géant. 1964) 120. «Les Etranges Etudes du Dr. Paukenschlänger» en Les Contes du whisky: 166. Malpertuis (Paris: Editions J'ai Lu. 1984). “Le Psautier de Mayence». “Le Grand nocturne», “La Trouvaille de Mr. Sweetpipe», “Mathématiques superieures» et "Le Tessaract». 
de normes humaines. D'autres, ressemblent à des espaces terrestres: des îles (Malpertuis, "L'Assiette de Moustier»), des maisons («Storchhaus» ou la «Maison des cigognes», Malpertuis, «L'Auberge des spectres»), des tavernes («Le Grand nocturne»). D'autres sont connus car ils appartiennent à la tradition chrétienne, comme par exemple l'enfer, l'au-delà et le purgatoire (sorte de plan intermédiaire où les âmes attendent le jugement $).{ }^{8}$ Cependant ceux-ci diffèrent de la vision imposée par l'imagerie populaire car ces sont des mondes tout aussi réels que l'espace terrestre. Ce n'est plus l'irrationnel qui nourrit le récit fantastique mais des formules algébriques. Elles structurent ces espaces hypergéométriques et imposent leur existence. L'introduction des mathématiques comme source génératrice de sa cosmogonie donne la possibilité à l'auteur non seulement de renouveler le répertoire de la littérature fantastique mais aussi d'opérer des variantes sur des thèmes traditionnels, dont surtout celui du pacte avec le diable («Mondschein-Dampfer» en Les Contes du whisky). En effet, les personnages confrontés à ce problème, plutôt que d'invoquer la bienveillance divine pouréchapper au pacte, ont recours à des axiomes mathématiques qui leur permettraient, selon Jean Ray, de s'évader sur un autre plan de l'espace où Satan ne pourrait plus les atteindre. Dans le cas des tueurs qui désirent fuir la justice humaine, le recours est assez semblable. Ils ne prient plus Dieu dans l'espoir d'être sauvés mais utilisent leurs connaissances mathématiques pour faire disparaître les évidences dans un autre monde. Grâce à ce savoir approfondi. les hommes peuvent même interférer dans l'univers divin car cette connaissance dévoile pratiquement tous les secrets de Dieu.

Il y a en outre dans cet univers intercalaire une abolition du temps et parfois même de l'espace. En fait, certains plans de l'hyperespace se présentent comme une sorte de «cristalli- sation» du temps où il n'y a plus de passé, de présent ou de futur. Le temps y est cyclique. Ainsi pour certains personnages, leur aventure fantastique

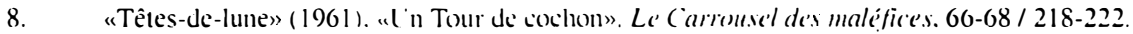


n'est qu'un éternel recommencement, les mêmes événements se reproduisant sans cesse. C'est l'expérience vécue par les dieux grecs dans Malpertuis et par A. dans le manuscrit français de «La Ruelle ténébreuse». Ce thème du temps cyclique est admirablement exploité à travers toute l'œuvre de l'auteur belge grâce à des motifs tels que la réincarnation des dieux, ou encore la présence des monstres ancestraux. ${ }^{9}$ Les fantômes, eux aussi, reviennent inlassablement hanter les lieux et les êtres humains de cet univers si particulier.

Loin de rejeter la thématique canonique, le conteur belge l'intègre parfaitement à son monde intercalaire en lui accordant une nouvelle signification, une dimension plus vaste. Tous les motifs et personnages classiques des récits fantastiques tels que les loupsgarous, les vampires, les fantômes, les monstruosités ancestrales, les dieux... acquièrent une autre identité et justification. Ils forment, sans contredit aucun, partie intégrante de cet univers au même titre que l'être humain. Une image plus moderne de ces êtres en résulte et l'auteur réussit une démystification totale. Le monstre devient moins cruel, plus humain; l'homme, par contre, est à ses yeux plus monstrueux. Il y a un constant chassé-croisé entre les deux: les bourreaux deviennent victimes, les victimes bourreaux. Un excellent exemple est celui de Tomas Wade qui souffre d'avoir perdu son fils à cause de Gilchrist, un homme méchant et sans scrupules. Il a fait couler le bateau où travaillait son garçon. Gilchrist sera transformé en araignée par un justicier de l'au-delà. Alors Tomas Wade va s'acharner à le faire souffrir. Il va le torturer physiquement et psychologiquement devenant ainsi un monstre de cruauté.

Ainsi l'homme peut, à n'importe quel moment, affronter ces créatures traditionnellement conçues comme étant beaucoup plus

9) Malpertuis: "Je cherche Mr. Hazenfraz", Les derniers Contes de Camterbury: A'ures Complètes. Tome 3. 52: “Hecates et Mr. Ram» (1952): Les comtes noirs du Golf. 188: "Têtes-delune" en Le Carrousel des maléfices. 68. "Dans le Marais du Fenn" en Les. Comtes du whisky: "La Croisière des ombres». 59. «La Bête blanche» (16.3-164); "L'Histoire du Wûlkhé» en $L$ t Grand Nocturne: "Le Uhu» en Les derniers Contes de Canterbury: Qurres Complètes. Tome 3.52 . 
fortes. Leurs forces s'équilibrent. Il peut les vaincre, les réduire en esclavage, les apprivoiser ou encore punir les morts en les soustrayant à la justice divine grâce à des grimoires (livre de sorcellerie) tout puissants. Ce rapport entre les entités non humaines et les hommes constitue l'un des points forts de son œuvre. L'homme n'est plus dominé, il n'est plus l'esclave et peut même devenir, grâce à ses connaissances, le maître du cosmos car il n'est jamais, comme dans les autres écrits fantastiques, écrasé d'avance par l'au-delà (les mondes parallèles). Quelle que soit la situation, il se bat pour survivre. L'auteur souligne de même la monstruosité dont fait preuve l'homme, défaut qui le met sur un plan d'égalité avec les créatures des mondes parallèles. Rien ne l'émeut, rien ne le choque. Ainsi, avec ces qualités ou ces défauts qu'il attribue à ses personnages, Jean Ray impose une nouvelle conception du héros dans la littérature fantastique, ce qui confère à son œuvre un caractère très moderne. En général, dans la littérature fantastique, les protagonistes des expériences insolites, surnaturelles, sont dépassés, anéantis par des événements qu'ils ne peuvent pas cerner ou comprendre, car ils n'entrent pas dans leur cadre de pensée. Ils succombent donc à la folie ou à la mort parce qu'ils sont incapables de réagir. Il en va autrement pour les personnages campés par le conteur belge car ce sont des hommes qui ne refusent jamais le combat. Ils sont, certes, déroutés, terrorisés, au début de leur expérience. Mais très vite, ils abandonnent toute raison raisonnante pour se laisser guider par leur intuition. Qu'ils soient sur un autre plan ou qu'ils affrontent des créatures étranges, ils attendent, scrutent, cherchent à percer le mystère, à trouver la clé de l'énigme. Lorsque l'homme a maîtrisé sa peur, que son esprit se libère du carcan de sa pensée cartésienne, il se laisse guider par son instinct et peut alors se battre. Il trouve les ressources nécessaires pour vaincre ces monstres. Quand ils découvrent les lois les régissant, ils se battent jusqu'à la mort s'il le faut. En faisant appel à leur sang froid et à leur savoir («La Ruelle ténébreuse», «Le Psautier de Mayence»; «M. Wohlmut et F. Benschneider», «La Vérité sur l'oncle Timothéus», 
«Un Tour de cochon», Malpertuis). Ils ont les mêmes chances de réussite («Le Psautier de Mayence»). Jacques Van Herp le résume judicieusement: «l'au-delà n'est qu'un adversaire comme tant d'autres: puissant, redoutable, mais toujours à la mesure de l'homme». ${ }^{10} \mathrm{Ils}$ combattent ces forces fantastiques avec des armes humaines. La femme prend, dans son œuvre narrative, une autre dimension qui va de pair avec celle des mondes intercalaires et qui donnera tout son sens aux éléments malfaisants de son univers. Elle est, aux yeux du conteur, un monstre de cruauté et d'égoïsme, de frivolité et de froideur. L'écrivain le manifeste dans plusieurs de ses contes où la femme est une redoutable criminelle comme Lady Honnybingle, en La Cité de l'indicible peur, ou «Les trois petites vieilles sur un banc», entre autres. " La frivolité est, d'après Jean Ray, l'un des plus grands défauts de la femme qui provoque la perte de celui qui l'approche. Il le confirme dans le manuscrit français de «La Ruelle ténébreuse» car le personnage vole dans l'impasse de la quatrième dimension afin de pouvoir combler les caprices de la femme quil aime. Anita est en effet très exigeante et cupide. Les êtres malfaisants sont souvent des femmes. Elles sont rusées. Telles des araignées, elles préparent patiemment leur piège en endormant la méfiance des hommes qu'elles convoitent. La femme est une habile manœuvrière qui s'amuse à dérouter les hommes pour mieux les contrôler. Les pouvoirs télépathiques de Marfa Dolpack, la sorcière ${ }^{12}$ lui permettent de lire les pensées des gens et d'agir en conséquence pour capturer leur attention. Fine psychologue, elle joue sur leurs faiblesses pour les faire agir dans son intérêt. Frivole et avide, elle n'a aucun scrupule à

1(). Jacques Van Herp. "L'univers de Jeain Ray». Les Cahiers de L'He'me (Paris: L'Herne. 1980) 217.

11. La Cité de l'indicible peur (Verviers: Marabout Géant. 1943): "Les trois petıtes vieilles sur un banc" en Le Carrousel des maléfices. 197: "Le Grand nocturne» (1942). Malpertuis, "La Clergesse parie» et "Le Chat assassiné» (1944). "La Sorcière» (1952) 165-181. "Les Gens célèbres de Tudor Street» (1955) 182-192. "Le Tessaract» (1956) en Le Carrousel desimaléfices: Less contes noirs du Goif.

12. «La Sorcière» en Le Carrousei des maléfices. i69-170. 
se servir des hommes; c'est pour cette raison qu'elle se laisse séduire plus facilement par les forces ténébreuses émanant de certains univers parallèles, notamment l'enfer. Monstre de cruauté dans la vie, elle se transforme aisément en symbole d'épouvante. Qu'on en juge à propos de la métamorphose subie par Hellen Kranert dans «MondscheinDampfer». En effet, femme superficielle elle pousse son amant à assister à une fête sur la Mögelsee où celui-ci rencontre Lucifer. Libertine, elle apparaît et disparaît avec des hommes jusqu'au moment où elle se dispute avec son amant et tombe à l'eau. Croyant l'avoir perdue, il signe un pacte avec Satan. Lorsqu'il la reverra, elle est devenue «diablesse». Sous l'apparence extérieure d'un corps féminin vibre souvent une créature abominable que des forces occultes font survenir. Qu'elle séjourne sur le plan terrestre ou réside dans un autre monde, qu'elle soit esprit des ténèbres, simple mortelle comme Hellen (ou déesse), elle apparaît terrible entre toutes les créatures, assoiffée de souffrance et de sang. Elle attaque avec fureur celui qui ose la réveiller, la provoquer, l'arracher des ténèbres de l'enfer où elle séjourne. Tel est le cas d'Alecto dans Malpertuis.

\section{Renouvellement de la thématique classique}

Jean Ray imprègne, d'une touche très personnelle la thématique classique qu'il développe. En tout premier lieu, parce que tous les thèmes sont liés aux espaces intercalaires. En effet, les espaces traditionnellement maléfiques (l'enfer, le purgatoire, l'au-delà, les univers des dieux) conçus auparavant comme des concepts abstraits selon des préceptes religieux sont, pour l'écrivain, des plans de la Quatrième dimension superposés ou parallèles à l'espace terrestre. De ce fait les thèmes légendaires teìs que la magie, la maison maudite, entre autres, subissent une véritable métamorphose. La magie n'est plus une simple conjuration des esprits, de Satan lui-même en quête de pouvoir mais plutôt un moyen de communication entre les différents plans parallèles; elle est une arme permettant de conquérir ces univers et les êtres y habitant ou d'emmener contre leur gré des esprits dans 
d'autres plans intercalaires. Grâce aux objets maléfiques comme les grimoires, les boules de cristal, les miroirs, les traités de magie, ${ }^{13}$ l'homme peut non seulement voler le diable, Dieu lui-même, mais aussi dominer des démons malfaisants, les anéantir, faire revenir les fantômes dans les mondes intercalaires. C'est le cas du fantôme de Marie Beer qui vient rejoindre Théodule Notte dans le plan du Grand Nocturne.

La maison maudite ${ }^{14}$ elle-même n'a plus de connotation maléfique au sens propre du terme. Elle constitue, pour le nouvelliste, un plan intermédiaire entre les divers univers. Elle remplit différentes fonctions selon les contes ou les nouvelles. Elle peut être un point de rencontre, de convergence entre ces mondes intercalaires ou devenir une prison, un piège ou encore un champ de bataille où s'affrontent les créatures des espaces hypergéométriques (l'être humain, le diable, les démons, les dieux, les monstres).

L'évolution des thèmes légendaires tels que la sirène, le loupgarou, le vampire, le diable, la mort entre autres est lente. C'est le cas de la femme-vampire. Dans son tout premier conte «Le Gardien du cimetière» ${ }^{15}(1920)$ l'écrivain reste fidèle à l'image promue par le récit fantastique car il nous la présente cruelle et sanguinaire. En 1928 dans la nouvelle «La Ruelle ténébreuse», il introduit des petites nouveautés en ce qui concerne le caractère psychologique du personnage. En effet, la femme-vampire reste toujours aussi bestiale mais elle peut être apprivoisée. Il rajeunit le sujet en lui donnant une coloration différente. En fait, la femelle-vampire n'est plus un être humain devenu sauvage mais une créature habitant une impasse intercalaire et qui peut acquérir des sentiments humains comme la peur ou l'attachement. Enfin, en 1944 dans le conte «Dieu. toi et moi» ${ }^{16}$ le changement est complet. La

13. "Dürer l'idiot», «Le Grand nocturne»: "Mondscheın-Dampfer» et «Le Bout de la rue».

i4. «Maison à vendre» en Le Livre des fantômes. Saint-Judas-de-ia-Nuit (Verviers: Marabout. 1966) 28: «Le Grand nocturne». "L'Auberge des spectres». «Storchhaus» ou ia "Maison des cigognes», Maipertuls.

15. En Les Comres du whisky. "La Croisière des ombres".

16. En Nomeani Comtes. Qinres Complètes. Tome 3, 168. 
vision est très moderne: ce n'est plus une vieille noble mais une jeune femme menant une vie simple. Elle a des principes moraux et souffre de sa condition. Elle est aux antipodes du personnage de son premier conte.

D'autres personnages du répertoire fantastique évoluent beaucoup plus vite, phénomène dû surtout à une conception particulière. Parmi eux, les plus remarquables sont la mort ${ }^{17}$ et le diable. L'image qu'il exploite correspond en partie à son acquis culturel. La mort n'est nullement effrayante pour les Flamands dont l'auteur est un digne ressortissant. Elle est plutôt une figure très familière avec qui il est possible de jouer aux cartes, de l'enivrer. Les premiers contes de Jean Ray «Le Dernier voyageur» (1929) et «Le Bout de la rue» (1931), respectent dans les grands traits la version classique. La mort y est décrite, surtout dans le premier conte comme étant un être invisible et de ce fait, elle est beaucoup plus effrayante. Dans le deuxième conte, son appartenance aux univers intercalaires est déjà chose résolue. La mort n'est pas en fait une fin en soi mais implique un passage dans la quatrième dimension, l'au-delà n'étant qu'un desplans del'hyperespace. Dès 1943 («Le Cimetière de Marlyweck» et «Le Miroir noir»), la personnalité de la mort commence à se définir. Elle devient plus humaine, elle est capable d'éprouver des sentiments tels que la rage et peut même être aussi têtue que certains hommes. Par la même occasion, ses attaches avec les uni vers parallèles sont confirmées. Elle se matérialise tout d'abord sous la forme d'une statue tout en gardant les attributs classiques, le sablier et la faux, puis évolue pour devenir en 1944 («La Vérité sur l'oncle Thimothéus») un homme en chair et en os. Elle est le double conforme des êtres humains avec leurs défauts et certaines de leurs qualités. Il y a cependant une différence de poids, son énorme pouvoir. Même les dieux la craignent.

17. «La Vérité sur l'oncle Timothéus» en Le Lirre des fantômes. Saint-Judas-de-la-Nuit, 225: «Le Cimetière de Marlỵweck". "Le Dernier voyageur». "Le Miroir noir» en Le Grand Nocfurne. 23.3. 247. 313. "Le Bout de la rue" en Les Contes du whisky. 
Le diable ${ }^{18}$ lui aussi, emprunte le même parcours. Jusqu'en 1944 il garde son image légendaire d'être cruel et tout puissant, d'incarnation du Mal qui provoque une peur indescriptible. Ainsi que la tradition le dépeint, ce serait une entité abominable aux mains gigantesques et difformes, avec une queue de dragon et un masque biscornu auréolé de soufre et de phosphore. Cependant, la même année l'auteur en présente une autre version. Sa descente aux enfers, si l'on peut ainsi s'exprimer, commence. En effet, il n'est plus un bourreau mais une victime. Il souffre comme tout être humain de la solitude. Il souhaiterait pouvoir ressentir des émotions telles que la joie, l'amour, la tendresse. Dès 1958, il commence à perdre tous ses pouvoirs. Il ne provoque donc plus la peur comme chez les autres auteurs. Il devient aussi médiocre que la mort et que l'homme luimême. Sa déchéance est si profonde qu'il se trouve au chômage parce que les gens ne croient plus en lui. Le diable n'est plus qu'une pauvre créature qui partage la misère des hommes. Pour Jean Ray, le diable est un esprit fraternel à qui une mauvaise réputation a été faite, surtout au Moyen Age, pour le rendre responsable des crimes commis par les hommes. Ce serait une forme, une force quasi familière, qui se présente sous différentes facettes. Mais il va encore plus loin dans Malpertuis et dans la plupart de ses contes consacrés au diable, où il se moque des peurs ancestrales éprouvées envers les dieux ou envers Satan lui-même. Il a une tendance certaine à désacraliser le divin et à démythifier l'image du diable (objet des peurs irraisonnées) et du maléfique en général car, d'après l'auteur, seule la mort est à craindre. Pour nous le prouver, il met en scène dans Malpertuis les toutpuissants dieux grecs dominés par un simple mortel qui les affronte avec de la magie noire. Il affirme également que les dieux tout comme

18. “Mondschein-Dampfer». «Dürer. lıdiot» et «Le Bout de la rue»: «Le Grand nocturne», «Maison à vendre» et "Reid Unthank» (1944): "Histoire sans titre» en Le.s Caluer de L'Herme (Paris: L'Herne. 1980) 391, "Unn Tour de cochon» et «Passez à la caisse» (1958): «Bonjour M. Jones» (1959) en Le Carrousel des maléfices, "Le Banc et la porte» et Saint-Judas-de-ia-Nuit (1960): "L'Envoyée de retour» (1961) en Le Carrousel de's maléfices. 
le diable doivent leur existence aux hommes qui les ont créés. Ces derniers peuvent donc les détruire à tout moment. Ce n'est pas l'homme qui doit craindre la colère des dieux mais les dieux celle des hommes.

Comme on peut le constater, ces thèmes se prêtent à un véritable changement. En humanisant la plupart de ces personnages réputés malfaisants, Jean Ray modernise leur conception. Il confirme ainsi son postulat selon lequel l'homme est l'égal de ces êtres légendaires ou folkloriques. Pour les affronter l'être humain recours, en tout premier lieu, à la logique, à l'esprit cartésien, à la science et aux mathématiques.

Dans les contes et les nouvelles du conteur belge, les atmosphères brumeuses règnent en maître absolu. En fait un trait caractéristique de son œuvre réside dans les atmosphères oppressantes qu'il crée afin de provoquer la peur à ses lecteurs. Les événements étranges y sont à peine ébauchés. Seul compte le déchaînement des éléments naturels qui engendreront l'apparition surnaturelle. Ainsi que l'infère Jean Delaet, le nouvelliste «use habilement des sensations confuses que nous ressentons tous dans le brouillard et l'obscurité, devant l'eau ou dans le vent, le long des quais un soir de pluie». ${ }^{19}$

Les éléments naturels ont eux aussi une importance capitale dans son œuvre. En fait, ils ne sont plus un élément du décor. De même que les créatures malfaisantes, ils acquièrent des caractéristiques très humaines où la cruauté prime. Ils sont les complices des êtres des espaces intercalaires. Grâce à leur accumulation, à leur multiplication, l'écrivain crée des effets aussi bien visuels qu'auditifs pourembrouiller non seulement l'esprit du héros mais aussi celui du lecteur. Un climat d'apocalypse règne. La démesure que l'auteur y laisse apparaître captive leur attention au point qu'ils subissent les événements surnaturels.

Les ténèbres sont le véritable univers du conteur. Tout y est noir. L'eau n'y est jamais cristalline, les marécages sont profonds, trompeurs 
tout comme les miroirs aux pouvoirs maléfiques; ils constituent en somme des voies d'accès conduisant vers l'horreur et permettent à l'épouvante de s'introduire dans l'univers terrestre. L'essence même de sa création demeure dans la «Grande Peur» qui hante toutes les créatures de sa cosmogonie. Le «fantastiqueur» le confirme ainsi dans la préface qu'il écrivit pour Les Chemins étranges de son ami Thomas Owen: «La Peur est d'essence divine, sans elle les espaces hypergéométriques seraient vides de dieux et d'esprits». ${ }^{20}$

Jean Ray considère ce sentiment d'inquiétude comme une fin située «au bout de la raison, de l'entendement, de la compréhension». Aussi symbolise-t-il, pour certains de ses confrères, le véritable poète de la peur car elle est toujours présente dans son univers rempli de présences innommables.

Évoquer en quelques lignes tous les traits qui font de l'écrivain belge l'un des maîtres incontestés de la littérature fantastique n'est pas aisé. J'essaierai donc d'ébaucher certains aspects essentiels qui permettront de mieux appréhender son œuvre.

\section{La structure de ses récits}

Jean Ray n'a pas seulement renouvelé la thématique fantastique mais a marqué de son originalité sa structure qui est à l'image de son univers fantastique tant au niveau de la composition du récit qu'au niveau de la technique narrative. Tout comme ses espaces hypergéométriques, ses récits se présentent sous d'innombrables facettes, ses compositions se multiplient et se compliquent à plaisir pour mieux traduire ces mondes parallèles qui forment l'axe central de sa création ainsi que le démontre la comparaison de la structure de son univers fantastique et de la nouvelle «La Ruelle ténébreuse» ci dessous.

Ce parallélisme existant entre la conception de son univers et la structure de l'œuvre est explicite dans nombreux contes et nouvelles 


\section{STRUCTURE DE «LA RUELLE TÉNÉBREUSE»}

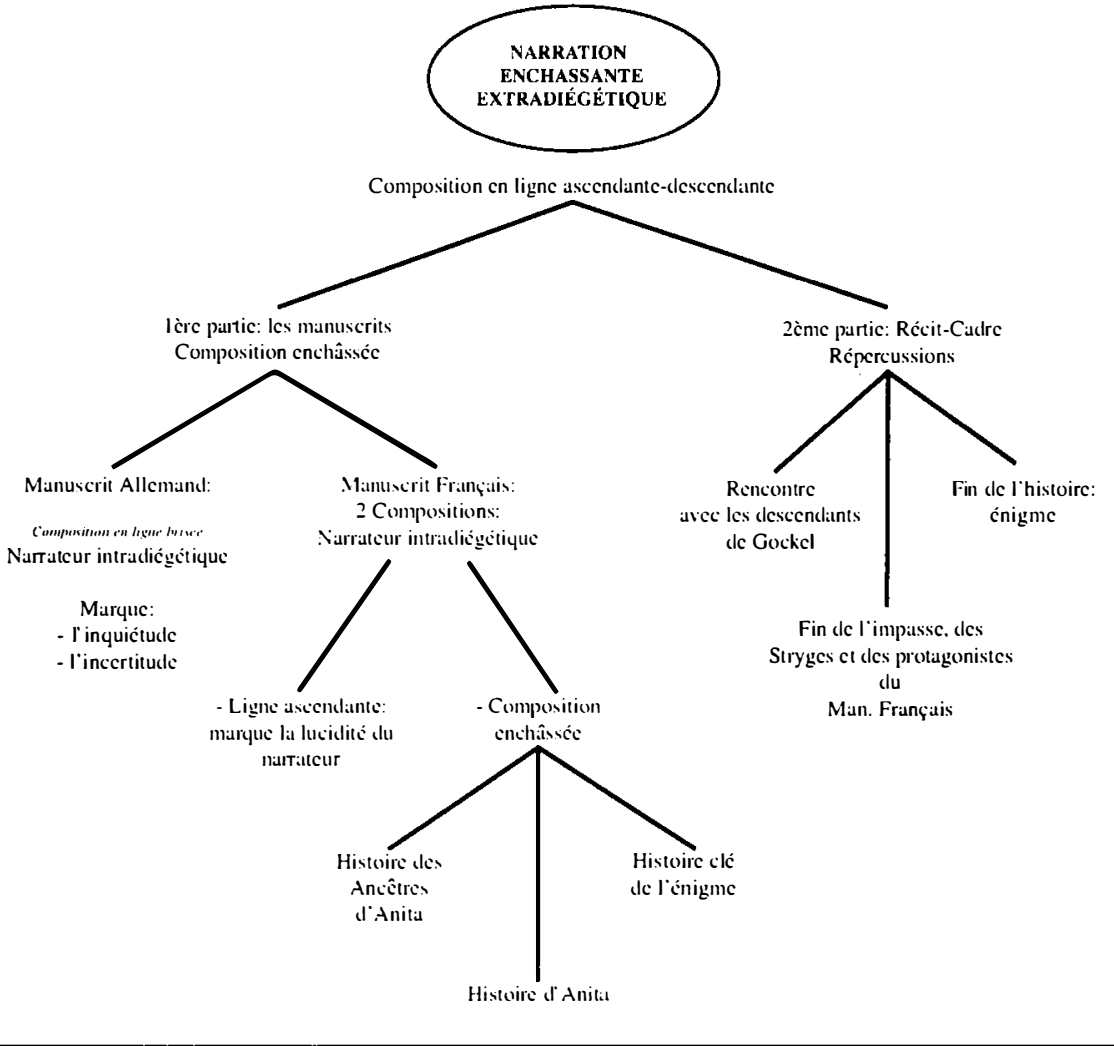

parmi lesquels ses chefs d'œuvre Malpertuis, «Le Psautier de Mayence», «Le Grand nocturne» et «La Ruelle ténébreuse».

L'analyse de cette dernière permettra de mieux comprendre le génie de cet écrivain dont la composition narrative est le reflet de ses mondes hypergéométriques.

Cette nouvelle est composée d'un récit cadre et de deux manuscrits présentant des histoires parallèles et liées entre elles. En 
effet, elle exploite le thème de la quatrième dimension car les protagonistes découvrent des plans intercalaires. Le héros du manuscrit français (A. Archipêtre) pénètre dans une impasse intercalaire accidentellement lorsqu'il est bousculé par une calèche. Cependant sa découverte est le fruit d'une observation scientifique. Il en va autrement pour l'héroïne du manuscrit allemand qui est emmenée dans la ruelle par la créature invisible qu'y habite. L'auteur présente deux conceptions complètement opposées du même espace parallèle. Pour l'héroïne cet espace est une petite maison paisible où elle a retrouvé la sécurité. Par contre pour le héros du deuxième manuscrit c'est l'antre de créatures sanguinaires qui pourraient aisément être confondues avec des démons ou des vampires assoiffés de sang:

La Bérégonnegasse et ses petites maisons ne sont qu'un masque, derrière lequel s'abrite, je ne sais quelle horrible face... L'escalier finissait sur un gouffre creusé à même dans la nuit et d'où montaient de vagues monstruosités. ${ }^{21}$

Il reprend, par la même occasion le thème de la Maison maudite qu'il présente comme étant un plan intermédiaire, un point de rencontre ou de passage entre les différents plans de la quatrième dimension.

La notion de la contraction du temps et de l'espace y est abordée sur deux plans: d'abord sur celui de l'histoire. En effet le héros évolue dans un espace qui se reproduit à plaisir formant une sorte de labyrinthe où il exécute toujours les mêmes actions.

Je vole éternellement dans une même maison, dans les mêmes circonstances, les mêmes objets ... Une courbe, trois petites portes jaunes, un bouquet de viornes, puis un nouveau coude, et puis réapparaissaient les trois petites portes dans le mur blanc. $^{22}$

21. "La Ruelle ténébreuse», 165.

22. Malpertuis. 158-161. 
Sur le plan structurel, cette cristallisation est suggérée dans le manuscrit allemand par la reprise de la même séquence narrative sept fois. Tout est un éternel recommencement car les disparitions sont marquées par les mêmes éléments. Celles-ci débutent dans une atmosphère de tension qui sera menée au climax par des bruits indéfinissables («bruit épouvantable», «bruit indéfinissable», «conques géantes»). Ils annoncent l'apparition de l'épouvante et la disparition des personnages qui ne sera jamais élucidée maintenant ainsi la tension initiale. Elles alternent avec des moments d'accalmie ponctués par des commentaires, des opinions personnelles, des interpellations au lecteur. En outre, il confirme son postulat de la Quatrième dimension en insérant parmi ces séquences narratives deux séquences intercalaires qui montrent le parallélisme existant entre les événements qui se passent à la maison et ceux subis par les personnes dans la ville. Par ailleurs la combinaison ou superposition d'histoires dans un même récit-cadre matérialise en quelque sorte ses univers intercalaires. Le lecteur implicite exécute en quelques lignes un voyage dans le temps qui le fait remonter du présent au passé ou le projette vers le futur tout naturellement grâce à succession d'analepses et des prolepses. De cette façon, il schématise la cristallisation du temps. Aucune incohérence ne se glisse ni dans le fond ni dans la forme. On peut dès lors affirmerque son fantastique impose à l'esprit du lecteur une rupture par rapport aux connaissances acquises ou par rapport aux points référentiels de son existence; ainsi que, d'un point de vue strictement littéraire, c'est également vrai par rapport à la linéarité dans la lecture d'un récit.

S'il est vrai que Jean Ray décuple les effets, accumule les personnages qui évoluent d'un extrême à l'autre, il a su se servir d'une structure du récit qui captive complètement le lecteur. Il combine à merveille le principe de concentration et la polyphonie narrative propres à des écrivains tels qu' Allan Poe et E. Hoffman.n. En effet, il utilise une construction par paliers ${ }^{23}$ composée de paragraphes

23. Ces paliers peuvent parfois être numérotés en chapitres ou porter des tıtres comme dans le cas de Malpertuis. 
généralement brefs séparés par des espaces ou des symboles. «La Ruelle ténébreuse» compte 26 paliers superposés, repartis inégalement entre le prologue (récit-cadre) où le narrateur raconte la découverte des deux cahiers qui semblent narrer deux versions différentes d'une même aventure fantastique, le Manuscrit allemand ( 8 paliers), le Manuscrit français ( 15 paliers) et la conclusion (2 paliers).

Dès le départ, tout est mis en œuvre pour capter l'attention du lecteur. Dans cette nouvelle l'écrivain débute son récit par la fin de l'histoire créant ainsi une atmosphère de suspens qui rendra le lecteur implicite prisonnier du texte. En fait l'histoire du manuscrit allemand impose une énigme: celle de la disparition inexpliquée des personnes dans la ville d'Hambourg qui vit dans la terreur. Ce mystère sera éclaircit par le deuxième manuscrit avec la découverte de l'impasse de la quatrième dimension où résident des créatures terrifiantes. En réalité, la première commence peu avant la fin de celle qui est contée dans le manuscrit français.

Pour captiver le lecteur, Jean Ray déploie, dès l'abord, l'une de ses principales techniques d'écriture: la concentration. En quelques lignes, il présente le décor, les personnages, l'atmosphère, le caractère insolite des histoires en amorçant le récit ${ }^{24}$ par une remarque où le narrateur établit la nature épouvantable de l'événement en s'exclamant comme dans le prologue «Pour autant que la lumière puisse se faire sur cette histoire qui paraît hantée des pires forces hostiles !» ${ }^{25}$

Parfois l'auteur commence la narration par un préambule introduit par une phrase énigmatique comme celle prononcée par A. Archipêtre: «Je suis à présent édifié» s'emparant ainsi de l'attention du lecteur dès la première ligne ou bien en confirmant, l'atmosphère de terreur qui va régner dans son histoire (deuxième ligne). C'est le

24. Un préambule qu'il introduit par une remarque philosophique ou par un incipit où le narrateur témoin établit la nature épouvantable de l'événement ou bien par un jugement du hèros sur lui même qui. malgré son caractère négatif, marque une certaine honnêteté de sa part et donne par ce fait de la crédibilité à son récit.

25. "La Ruelle ténébreuse». 118. 
cas de la narratrice du manuscrit allemand. Par la suite, il l'entraîne directement dans le monde du personnage. Il le plonge d'emblée dans son existence, le familiarise d'emblée avec la réalité de celui-ci et dans une atmosphère paisible qui endormira toute suspicion de sa part. En effet, l'héroïne de la première histoire dépeint l'ambiance placide qu'elle partage avec ses amies malgré la tempête qui se déchaîne à l'extérieur. A. Archipêtre, lui, se trouve dans une taverne à discuter et à boire joyeusement avec un vieux cocher. Ce préambule permet à Jean Ray d'établir l'existence de la ruelle intercalaire. C'est un jeu savamment structuré qui lui permet de prendre au piège le lecteur implicite qu'il rassure à tout moment grâce à des remarques scientifiques ou à la qualité des témoins mis en scène qui assurent la véracité et la crédibilité de l'histoire. Dans ce cas le témoignage du plus vieux cocher de la ville est irréfutable. Pour écarter toute contestation possible il renchérit avec celui d'un étudiant. Le fait que le héros soit professeur n'est nullement gratuit. Tout est mis en œuvre pour éviter toute objection du lecteur afin de faire accepter son postulat de la quatrième dimension.

L'attention du lecteur est conquise au plus vite et le restera jusqu'au dénouement grâce à une composition extrêmement riche. ${ }^{26}$ Afin de concrétiser son univers fantastique il a choisi la construction par emboîtement où des histoires fantastiques sont enchâssées ${ }^{27}$ dans un premier récit ce qui lui permettra de développer dans chaque narration une courbe narrative différente en accordant à chacune une autre dimension, il leur donne ainsi leur expression maximale. Effectivement, chez lui cette composition se complique à plaisir. Il ne se contente pas d'imbriquer une seule histoire mais deux dans le récit

26. Cette composition la de la simple gradation ascendante jusçu'à la construction enchâssée en passant par les compositions en ligne ascendante-descendante et en ligne brisée. Ces courbes dramatiques sont aussi fréquentes les unes que les autres et illustrent parfaitement bien la conception de l'univers fantastique de l'auteur.

27. Ses plus grands chefs d'œuvres sont structurés par des récits enchâssés. C'est le cas de SaintJudas-de-la Nuit. «Le Psautier de Mayence», "Ĺe Bout de la rue», «La Présence horrifiante». "Maison à vendre». "L'Histoire de Marshall Grov'c». Malpe'rtuis. "La Ruelle ténébreuse». 
cadre sous la forme d'un journal intime et des mémoires. Mais il ne s'arrête pas à cet emboîtement car il déploiera au maximum toute son utilité stratégique en ce qui concerne la peinture de son monde fantastique dans le manuscrit français. Cette juxtaposition d'histoires dans le même récit s'accorde parfaitement avec sa conception des mondes intercalaires. Ainsi qu'il a été expliqué auparavant elle est composée d'un récit cadre qui présente deux manuscrits superposés qui se complètent et s'expliquent mutuellement. Ce récit cadre est structuré par une composition en ligne ascendante-descendante. ${ }^{28}$ Elle permet au conteur belge d'imposer à l'esprit du lecteur implicite l'hésitation propre au fantastique. Il est divisé en deux parties. La première qui contient les deux manuscrits mène le héros et le lecteur à l'événement insolite dans une progression savamment orchestrée tandis que la deuxième expose les répercussions et essaie de donner des explications rationnelles au phénomène. Celle-ci à son tour est composée de trois récits. D'abord le narrateur extradiégétique nous raconte sa rencontre avec les descendants de l'antiquaire Gockel, le deuxième explique la fin des personnages et le troisième les répercutions dans leur vie. Mais les théories émises dans cette conclusion ne sont pas convaincantes. Au lieu de rassurer le lecteur, elles provoquent chez lui un certain malaise. Le mystère, l'ambigüité demeurent, au lecteur de s'en sortir.

Chaque histoire insérée dans le récit-cadre possède sa propre courbe dramatique qui permet de traduire l'état d'âme des différents narrateurs-héros. Aussi, pour mieux comprendre cette nouvelle et ne pas se perdre dans les méandres de ses histoires, un tableau récapitulatif a été établi afin de guider le lecteur. Il est intercalé à la page 33. La première histoire «Le Manuscrit allemand» est articulée par une composition en ligne brisée ${ }^{29}$ qui marque bien le trouble, l'hésitation,

28. La composition en ligne ascendante-descedante place le phenomene surnaturei au caur de l'histoire. Parmi les contes les plus réprésentatifs, citons "L'Assiette de Moustier». "L'Hıstorre du Wûlkhé». "L'Homme qui osa», "La Sorcière». "La Tête de Monsieur Ramberger», "Le Dernier voyageur"

2). Non moins intéressante est la structure en ligne brisée où le phénomène surnaturel frappe à intervalles réguliers. souvent de plus en plus fort pour aboutir à la disparition du protagoniste. 
l'incertitude dominant la narratrice. En effet, elle est confrontée à la crise de la disparition de ses amies enlevées par des créatures sanguinaires. ${ }^{30}$ Ces disparitions alternent avecdes moments d'accalmie pendant lesquels la narratrice s'adresse directement au lecteur implicite pour l'éclairer quelque peu sur sa situation ainsi que pour porter des jugements sur l'attitude des autres personnages, sur leurs états d'âme, sur son propre trouble, sa perception de la situation. Les moments d'accalmie permettent à l'auteur d'opérer des ralentissements dans l'histoire. La tension qu'il a provoquée chez le lecteur est allégée grâce aux explications qu'il donne ou à l'atmosphère paisible qu'il crée. Son esprit ne se méfie plus. Jean Ray peut à nouveau le surprendre, l'effrayer tout comme le héroïne qui vit l'expérience. De cette manière, la courbe dramatique est parfaite: des moments de tension alternent avec des passages d'accalmie entre les différentes disparitions, entretenant ainsi l'attention du lecteur et l'incertitude propre au fantastique.

Quant au récit de la deuxième histoire, il combine parfaitement deux structures: la progression ascendante et la composition enchâssée sans que l'une interfère dans l'autre. Trois petites histoires se greffent au récit central: celle des ancêtres $\mathrm{d}^{\prime} \mathrm{Anita}^{31}$ où l'on explique ses origines et sa personnalité. L'histoire suivante, celle d'Anita renseigne le lecteur sur la relation que celle-ci entretient avec le protagoniste. Ce personnage est important parce qu'il entraîne le héros et le lecteur à s'aventurer dans la ruelle, c'est-à-dire dans l'impasse intercalaire.

Dans l'œuvre de Jean Ray. cette composition structure les récits des contes et nouveaux fantastiques tels que "L'Auberge des spectres», "Le Miroir noir», "Mondschein-Dampfer», "Dïrer l'idiot», "Le Cimetiere de Marlyweck". Ces deux derniers constituent les meilleurs exemples de sa technique. Dans ie conte “Le Cimetière de Mariyweck", la mort. représentée par une statue de bronze et par des tombes qui s'animent. se manifeste trois fois à intervalles réguliers. Une première fois. dans le cimetière de Marlyw'eck situé dans un plan parallèle de la quatrième dimension. puis dans le jardin du protagoniste. En effet, les tombes viennent de l'audelà pour saccager le jardin du héros. Enfin dans la dernière partie, la Mort vient l'emmener dans l'au-delà. symbolisé dans le conte par le cimetière.

30. «La Ruelle ténébreuse», 124-128.

31. Anita est ia femme dont ie héros est amoureux. 
Enfin la troisième dévoile la clé du mystère au héros, A. Archipêtre. Ces récits ne brisent en aucune manière la progression ascendante $\mathrm{e}^{32} \mathrm{de}$ l'histoire.

Cette dernière structure marque l'extrême lucidité du narrateur qui analyse chaque élément de la situation avec une précision quasi scientifique. La progression s'établit à partir de paliers - présentant chacun un élément de l'histoire - qui mènent le lecteur vers le point culminant de l'action, c'est à dire au moment de l'apparition insolite. Grâce à ces éléments superposés qui suggèrent remarquablement les plans juxtaposés de sa Quatrième dimension, l'auteur réussit un crescendo parfait qui retient l'intérêt du lecteur jusqu'au bout. Ceci permettra au lecteur de comprendre les événements surnaturels vécus par la narratrice du premier manuscrit. Le récit se termine parun retour au monde réel du narrateur et du lecteur. Ce procédé du récit enchâssé rend moins inquiétante l'histoire surnaturelle et permet au lecteur de mieux appréhender les événements insolites rapportés par le narrateur. Cependant pour le lecteur et le narrateur, un doute sur la réalité des expériences vécues par les protagonistes subsiste. Le narrateur va donc, dans la plupart des cas, chercher à éclaircir le mystère. Le lecteur, lui, est moins fortuné. Il devra trouver sa propre réponse...

Pour mieux dépeindre sa cosmogonie, il a inventé une écriture intercalaire $^{33}$ où se superposent ou s'imbriquent, à l'instar de ses mondes parallèles, différents modes d'écriture. Ses histoires font alterner différents modes d'écriture parmi lesquels la traduction du journal intime de l'héroïne du manuscrit allemand, les extraits de mémoire d'A. Archipêtre, des histoires personnelles, des descriptions, des récits explicatifs, des notes, des commentaires, des confessions, des témoignages, des dialogues rapportés entre autres.

\footnotetext{
32. La progression ascendante structure particulièrement des contes très courts comme "Le Singe», "La Maın de Goetz von Verlichungen", "Le Tableau». "Le Gardien du Cimetrìre". aınsi que la nouvelle Le Grand Nocturne.

33. Cette écriture intercalaire acquiert toute sa valeur et son utilité dans les nouvellés telles que Saint-Judas-de-Ia-Nuit. "Le Bout de la rue», "L'Histoire de Marshall Grove», "Maison à vendre", le roman Malpertuis.
} 
C'est sa grande force. L'accumulation des techniques lui permet de distiller dans l'esprit du lecteur implicite l'idée qu'il se fait du fantastique, une conception très moderne qui mélange harmonieusement la science fiction et les expériences surnaturelles. Il nous fait passer d'un univers à l'autre aussi imperceptiblement que ses protagonistes pénètrent ses espaces hypergéométriques. C'est l'un des points forts de sa technique et de sa modernité.

Cette diversité discursive déployée dans ses récits impose évidemment, une polyphonie narrative $\mathrm{e}^{34}$ où le narrateur joue un rôle essentiel. En effet la nouvelle «La Ruelle ténébreuse» en compte cinq. Le récit est structuré par plusieurs voix provoquant ainsi l'adhésion du lecteur implicite. Le récit cadre est transmis par un narrateur auteur ou intermédiaire. Il a pour mission d'organiser le récit, d'en assurer la continuité en articulant le passage d'un narrateur à l'autre, d'un événement surnaturel à l'autre, de guider le lecteur dans le labyrinthe du récit comme dans celui de l'espace hypergéométrique de l'univers fantastique de Jean Ray. Il rapporte les événements comme un simple fait sans s'impliquer dans le récit, supprimant de cette façon toute tension. Par ce fait, il assure un compte-rendu fidèle de la situation. Il commente les dernières lignes pour expliquer les mots énigmatiques du personnage dans le but d'éclaircir le mystère:

Je traduis les pages allemandes ... Ainsi se termine, comme coupé au couteau, le manuscrit allemand... Ainsi finit le manuscrit français ... Ainsi doivent écrire ceux, qui sur un bateau qui sombre, veulent confier un dernier adieu à une famille qui, espèrent- ils, leur survivra. ${ }^{35}$

Il est intéressant de constater que tous les narrateurs dans l'œuvre de Jean Ray sont polyvalents car ils peuvent à tout moment jouer

34. Cette poiyphome narrative aéploie toute sa force dans des contes tels que «Dürer l'idiot»: "Maıson à vendre»: «La Présence horrifiante» avec trois narrateurs, et le roman Malpertuis qui en compte hut.

35. «La Ruelle ténébreuse». 118. 1.39. 172. 
plusieurs rôles. C'est le cas des deux narrateurs-héros des manuscrits. Ainsi, par exemple, la narratrice-héroïne du manuscrit allemand dont la fonction principale est de transmettre l'événement insolite au lecteur implicite. Comme elle est le seul narrateur de l'histoire, elle reprend le rôle d'intermédiaire. Elle guide le lecteur pas à pas en décrivant le cadre, l'attitude des personnages, ses propres actions. Elle parsème son récit, ici et là, de réflexions concernant ses sentiments, ses sensations, sa perception des autres personnages ainsi que de leurs états d'âme. De cette manière, elle réussit à faire partager, au lecteur, le trouble qu'elle a ressenti lors des événements insolites qu'elle a vécus. Le fait qu'elle soit la protagoniste de l'histoire confère encore plus ·d'authenticité au récit. Bien qu'elle soit le seul narrateur, la polyphonie est assurée par l'emploi du dialogue rapporté qui alterne avec les descriptions faites par la narratrice. Le dialogue rapporté a pour but de rendre plus vivant le récit ainsi que d'exposer le point de vue des protagonistes de l'histoire et de dépeindre leurs traits de caractère à travers leurs répliques.

Le fait qu'il y ait deux narrateurs héros qui ont vécu la même aventure fantastique avec des conséquences et des perceptions dissemblables permet de mieux comprendre les différentes facettes de cette histoire. L'un présente les êtres de la quatrième dimension, l'autre le plan intercalaire lui-même.

Ces narrateurs-héros peuvent aussi prendre le rôle de narrateur rapporteur. C'est le cas d'A. Archipêtre qui relate l'histoire d'Anita, de ses ancêtres et celle de Mendell qui sont des éléments clés. Les deux premières expliquent l'intrusion du héros dans la ruelle intercalaire et justifient ses actions. La troisième élucide l'énigme de la disparition des personnes et matérialise en quelque sorte les êtres de ce monde parallèle.

Dans cette nouvelle l'épilogue est pris en charge par deux narrateurs-rapporteurs. L'un d'eux renseigne le lecteur sur le sort du héros du manuscrit français ainsi que sur les événements omis dans le récit. Le deuxième est chargé de la conclusion. Mais au lieu d'effacer 
toute ambigüité, il termine l'histoire par une réflexion angoissée qui renforce l'incertitude du lecteur quant à la nature rationnelle ou surnaturelle de l'histoire:

-Toutes les nuits elles sont là, les Choses, tu le sais bien, dit-elle, d'une voix basse comme un murmure douloureux. Elles assiègent nos pensées dès que le sommeil vient sur nous. Oh ! $\mathrm{Ne}$ plus dormir !... Elles reviendront toujours, tant que nous durerons et tant que durera cette terre de Malheur ! ${ }^{36}$

L'éloignement de ces personnages par rapport aux événements racontés dans les manuscrits et leur sentiment d'effroi à l'évocation des faits laisse perplexe le lecteur. De là son hésitation. Le lecteur est pris au piège. Il devient dès lors acteur car il doit lire, interpréter, chercher à résoudre des énigmes. Et pourtant, ce n'est pas toujours facile car l'habile conteur belge brouille toutes les pistes. Le lecteur s'identifie au héros et tout comme lui suit les événements dans l'attente, dans l'angoisse de ce qui va arriver, anxieux de connaître le dénouement. ${ }^{37}$

Souvent l'emprise du récit est prolongée au-delà de la fin et cela grâce à l'astuce de l'auteur qui termine ses histoires par des questions angoissantes comme celle de la narratrice du manuscrit allemand: «Où suis-je? Seule. Pourtant, tout ici est plein de tumulte..., une présence invisible mais effrénée est partout». ${ }^{38}$ Ou bien par des phrases énigmatiques, des cris, des appels au secours, des bruits, des exclamations, etc.: «Les Stryges! Les Stryges ! Les Stryges !». Au lecteur de trouver sa réponse, son explication!

36. "La Ruelle ténébreuse». 132.

37. Un excelient exemple de ce deroulement est ceiui du roman Malpertuis où ies documents présentés par le narrateur n'apportent au lecteur aucun éclaircissement sur les faits insolites. II les subit avec la même intensité. la mème incompréhension que le héros Jean-Jacques Grandsire. L'ambigüité persistc.

38. «La Ruelle ténébreuse». 132. 
Enfin, on peut affirmer que les narrateurs campés par l'auteur belge reflètent à merveille ses univers intercalaires comme l'atteste le schéma des espaces hypergéométriques de son univers et celui des narrateurs de «La Ruelle ténébreuse» inclus à la fin de cet article. Ainsi qu'il vient d'être démontré, ces narrateurs assument deux ou trois rôles parallèles. Ils sont à la fois narrateur-auteur ou intermédiaire, héros et témoins. Le nouvelliste déploie ainsi toute sa technique de la concentration. Quoi qu'il en soit et grâce à cette polyphonie narrative, l'auteur assure une parfaite compréhension de l'énigme car le phénomène surnaturel est cerné de toutes parts.

Dans la plupart de ses œuvres, l'auteur impose les paradoxes propres à sa conception du monde et plus particulièrement celle des Univers parallèles. Le lecteur se laisse ainsi prendre à son jeu éprouvant de l'incertitude à chaque moment.

L'incertitude provoquée chez le lecteur est palpable dans cette narration car le nouvelliste se plaît à remettre en questions les convictions du lecteur par rapport au bien et au mal, aux valeurs morales, aux victimes et aux bourreaux. Un excellent exemple est celui de Méta qui au début de l'histoire se présente comme une femme douce et fragile et devient à la fin plus cruelle que la créature invisible de la Quatrième dimension. Tout comme Tomas Wade le héros de «Irish Whisky», sa métamorphose est totale. Elle poursuit de sa haine la créature invisible qui a fait disparaître ses sœurs. Elle veut se venger. Par contre le monstre (le stryge, sorte de vampire des mondes intercalaires) qui tue ses sœurs se laisse apprivoiser par la narratrice et commence à éprouver des sentiments humains comme la tendresse, l'affection, l'inquiétude. Lorsque Méta veut frapper sa protectrice, il s'interpose pour la protéger mettant ainsi en péril sa propre vie. Le lecteur hésite à la fin de l'histoire Qui doit-on plaindre? Qui est la victime? Qui est le monstre de cruauté? Méta ou le stryge?

L'œuvre du fantastiqueur est marquée par une construction antithétique tant au niveau de ses personnages, de son style qu'au niveau de la structure elle-même. Cette technique permet au nouvelliste 
de maintenir l'ambigüité propre au genre. L'antithétique imprègne également sa composition. En effet si on compare les deux histoires, la première débute par la violence pour se terminer dans une atmosphère paisible tandis que la deuxième commence dans un climat rassurant pour finir dans l'horreur.

Cette technique marque aussi son style. Elle lui donne ses accents et ses couleurs propres. Il y a en effet dans ses écrits un usage fréquent des superlatifs, métaphores, d'images, qui se superposent ou s'entremêlent les uns aux autres dans un crescendo parfait afin de créer des atmosphères où l'horreur, la terreur suintent. Grâce à une langue imagée, il dépeint l'indicible, l'épouvante à partir des décors quotidiens imposant ainsi la démesure qui plonge ses récits dans la terreur.

Généralement Jean Ray, par des jeux de contraste du type silence / bruit, lumière / obscurité, crée une atmosphère de tension et de peur. Lecteur et héros sont ainsi tenus en haleine, la peur au ventre. Grâce à cette technique qu'il maîtrise à merveille, il réussit à tenir en éveil leurs sens de la vue et de l'ouie.

Il oppose constamment l'extérieur plongé dans l'obscurité et le froid parce que la tempête fait rage à l'intérieur qui baigne dans la lumière et la chaleur, le luxe douillet (la soie, le velours, le cristal) qui rassure autant les personnages que le lecteur. Cependant l'ambiance de fête n'arrive pas à effacer le danger des éléments naturels déchaînés qui laissent présager des événements terribles. En fait, pour l'auteur la rue est toujours ténébreuse et menaçante et la maison sécurisante. Dans le manuscrit français, par exemple, l'auteur confirme cette vision:

Tout cela baignait dans cette lueur ivoirienne des vitraux dépolis qui formait le plafond ...par une fenêtre grillée, je vis une courette ténébreuse, formant puits entre quatre murs immenses et moussus. ${ }^{39}$ 
Mais c'est encore un simulacre visant à endormir la raison du personnage et du lecteur car ces maisons ce sont des pièges.

Ainsi qu'il vient d'être expliqué, il oppose les personnages, inverse les rôles. Ceux-ci se métamorphose pour devenir leur antithèse. C'est un constant chassé-croisé entre-eux. Ainsi dans «La Ruelle ténébreuse», Méta Rückhardt, vieille fille douce, voit ses sœurs disparaître l'une après l'autre, tuées par un monstre invisible et cruel. Elle, par son caractère placide est une victime potentielle de la créature mais au fur et à mesure qu'elle vit sous l'emprise de la peur, elle devient dure, haineuse, terrible, sauvage, démoniaque. Cette proie apparente se transforme en un chasseur impitoyable qui guette, scrute chaque coin de la maison à la recherche de l'être qui la menace:

Méta veut se venger. C'est elle à présent qui guette les invisibles... Méta s'apparente elle-même à une sorte de spectre ricanant... Ce n'est plus le visage placide qui se penchait il y a quelques jours à peine, sur de la broderie aux soies éclatantes, mais une figure sauvage où brûle une double flamme de haine que parfois elle darde sur moi. ${ }^{40}$

Par contre, l'entité sanguinaire qui tue froidement ses victimes acquiert, peu à peu, des sentiments et des réactions humaines sous la bonne influence de la narratrice du manuscrit allemand qui la nourrit et la protège de la rage folle de Méta. Elle [l'entité] éprouve alors une tendre affection pour sa bienfaitrice. Ce n'est plus l'assassin qui massacre ses victimes froidement. Aussi, lorsqu'elle est découverte par Méta, elle la supplie, comme un être humain, d'épargner sa vie et celle de la narratrice. Au bout de sa mutation, le bourreau qu'elle était devient victime. Il y a entre Méta et ce monstre un chassé-croisé où ils deviennent l'opposé de ce qu'ils étaient au début de leur aventure. Voici comment le conteur marque les changements radicaux suivis par Méta et le Stryge. 


\section{Méta}

bourreau du Stryge (137)

masque démoniaque (137)

rire horrible (124)

figure sauvage où brûle la

haine (133)

s'apparente à un spectre

ricanant (133)

rage froide dans son regard

(130)

expression de colère (125)

douce, avec un visage placide

(122)

victime potentielle du Stryge victime de Méta (137)

\section{Stryge}

bourreau de Méta (124)

plainte douloureuse-

suppliante (134)

pleurs humains (134)

devient tendre (134)

a peur (135)

devient imprudent (136)

devient suppliant (138)

On retrouve, une fois de plus, dans ce roman le leitmotiv cher au conteur belge, celui du faux-semblant qui imprègne son œuvre.

D'une manière générale on peut certainement qualifier l'œuvre de cet étonnant «fantastiqueur» comme une littérature de transfiguration mentale qui bouscule les limites de la raison et qui insinue dans l'esprit du lecteur non seulement l'ambigüité mais aussi l'incertitude sur le monde réel car il impose une conception nouvelle de l'univers.

Cet article se veut une approche des Mondes parallèles dans l'œuvre de Jean Ray pour que ceux qui apprécient le genre fantastique puissent plonger dans son univers. D'autre part ce travail permettra-il peut-être que les réticents aient envie de faire incursion dans la littérature fantastique par le biais de cette double ouverture que sont les mondes parallèles. D'ailleurs ces mondes parallèles ou ces univers ne sont-ils pas un sujet à approfondir? Ces mondes intercalaires n'existentils pas dans le réel et l'irréel latino-américains? Ceux qui s'y connaissent ont la parole. Il serait souhaitable que cet article ait des échos pour enrichir le champ de recherche. 